\title{
Dermatological Classification Using Deep Learning of Skin Image and Patient Background Knowledge
}

\author{
Kittipat Sriwong, Supaporn Bunrit, Kittisak Kerdprasop, and Nittaya Kerdprasop
}

\begin{abstract}
Skin cancer is one of the most common human malignancies. It is a kind of skin diseases caused by abnormal growth of skin cells. Clinically, dermatological disease including skin cancer can be divided into many types. Treatment options for each type are varying depending on the prognosis of a disease. Type of skin disease or dermatological classification is an initial process of clinical screening. Traditional method of initial clinical screening requires a visual diagnosing by specialized expertise. In case the disease is classified as a type of skin cancers, it is a serious case of dermatological disease that should be treated promptly. Therefore, an automatic approach applied for this classification task is very useful. In this work, we propose an automatic method for skin disease classification using deep learning model of convolution neural network, or CNN. In order to increase the classification performance of $\mathrm{CNN}$, we employ both image data and background knowledge of the patient in the modeling process. The experimental results performed on a public dataset show that the $\mathrm{CNN}$ model can classify skin diseases with 79.29\% accuracy, while our proposed method to incorporate background knowledge of patient in the modeling phase can improve the accuracy up to $80.39 \%$.
\end{abstract}

Index Terms-Skin cancer, dermatological image classification, deep learning, convolution neural network.

\section{INTRODUCTION}

Skin cancer is one of the most common human diseases [1] [2]. Clinically, dermatological diseases including skin cancers can be divided into many types. Treatment options and prognoses for each type are varying widely depending on the type and the stage of the disease. The tradition clinical method to classify the type of skin disease uses visually diagnosis in a preliminary clinical skin cancer screening. This requires specialized expertise where the prognosis by dermatoscopy is followed by a biopsy, and histopathological examination.

Nowadays, machine learning techniques are intensively used to analyze the medical information. Generally, it is used to create the model for predicting what information will appear in the future or automatically classifying the existing medical data into a correct group or category. Deep learning (DL) is one of the most popular and state-of-the-art methods

Manuscript received July 11, 2019; revised October 6, 2019. This work was supported by Suranaree University of Technology (SUT), and the National Research Council of Thailand.

The authors are with the School of Computer Engineering, SUT, 111 University Avenue, Muang, Nakhon Ratchasima 30000, Thailand (e-mail: kittipat.re@gmail.com,_sbunrit@sut.ac.th,_kerdpras@sut.ac.th, nittaya@sut.ac.th). of machine learning. Principally, DL techniques utilize the basic learning method based on the famous artificial neural network (ANN) concept. But DL uses many more processing layers or steps than the ANN. DL methods are rapidly gaining much interest in the research and industry communities [3] on various tasks such as image recognition [4]-[6], speech recognition [7], [8], age estimation [9], prediction of mutation in DNA [10], [11], detection of subtype blood cells [12], and identifying metastatic of breast cancer from images of sentinel lymph node biopsies [13].

We consider applying DL that has been enhanced its performance with the background knowledge to extract useful features for better classify the skin diseases. After the review of DL and related work in Section II, we propose our research framework and a step-by-step detail in Section III. The experimentations and results are displayed in Section IV. We finally conclude our paper in Section V.

\section{PRELIMINARY AND Literature REVIEW}

\section{A. Deep Learning Method}

DL method is based on the artificial neural network (ANN) concept. The major extension is that DL uses many more processing layers than the ANN. Therefore, the training phase of DL takes longer time than the ANN. Despite the trade-off in computational time, DL gains popularity from its higher accuracy than the than ANN.

There exist many examples for accuracy improvement from moving from ANN modeling to DL. For instance, the work of Zhang et al. [14] reported that the application of DL method, called Joint Deep Learning Land Cover (JDL-LC), for land cover classification show overall accuracy improvement up to $89.64 \%$ and $90.72 \%$ for the Southampton and Manchester areas, respectively. These accuracy rates are higher than the ANN method using multilayer perceptron that can classify land cover types over Southampton and Manchester areas with $81.29 \%$ and $82.22 \%$ accuracy rates, respectively.

In general, DL method can be classified into four major types based on the network architecture [15]. These DL types are Unsupervised Pretrained Networks (UPNs), Recurrent Neural Networks, Recursive Neural Networks, and Convolutional Neural Networks (CNNs). Brief introduction of these DL types are as follows:

\section{- Unsupervised Pretrained Networks (UPNs)}

Example of this DL architecture is autoencoder [16]. An autoencoder is used to learn data coding scheme that is the best representative (called encoding) for a specific data set. This method can also be used for dimensionality reduction. The nature of this architectures is learning the input data 
features for generating output data features that is the same as the input features.

\section{- Recurrent Neural Networks}

This architecture was created to be used for sequential inputs with the time factor, which is the factor that causes the difference between the elements of the sequence. Simple examples of sequential inputs are video (sequence of images) and text (sequence of words). The main characteristic of recurrent neural networks is that the output of the previous node is the input of the next node. The famous type of this architecture is Long Short-Term Memory networks (LSTMs).

\section{- Recursive Neural Networks}

This architecture is like the recurrent neural networks in the sense that it can deal with variable length input. But there is a minor difference in that the recursive neural network is more like a hierarchical network with no time aspect associated with the input sequence. With recursive neural network architecture, the input data has to be processed hierarchically in a tree model.

\section{- Convolutional Neural Networks (CNNs)}

One of the most famous DL methods is Convolutional Neural Networks (CNNs) [17]. The main goal of CNNs is to learn image patterns and characteristics for the purpose of recognition and classification. The images used as input data of the CNNs can be human faces, street signs, construction sites, and any other aspects of visual data. CNNs use a set of images for training appropriate parameters in the network architecture. Basically, there are three main layers in the CNN network.

1) Convolutional layer. It consists of a group of nodes used for extracting important features from the images. This layer employs many of filters to operate on input images.

2) Pooling layer. It is a layer that is normally applied after the convolutional layer. The main advantage of this layer is to reduce the spatial dimension (width, height) of the input data that will send forward to the next convolutional layer.

3) Fully-connected layer. The nodes in this layer are fully connected to the output from the previous layer. This layer is usually used as the last step of CNN network before the output layer.

The CNN architectures are based on the pattern of layers, as demonstrated in Fig. 1.

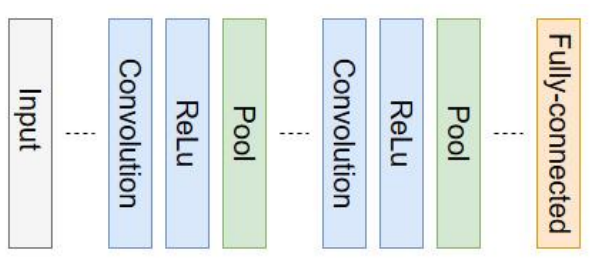

Fig. 1. General CNN architecture.

Implementing $\mathrm{CNN}$ architecture for classification can be done by creating a new deep network, or in other words training from scratch. With the train-from-scratch scheme, we must firstly determine the size of the images used as input. Then, configure the appropriate number of convolutional layer, pooling layer, and fully-connected layer. This method requires thousands of millions of images to train, which results in having to spend a long time in training.

A better learning scheme is to use the pre-trained $\mathrm{CNN}$ models for classifying a new set of images. The pre-trained CNN models are the ones that had been already trained on more than a million images and can classify the category of new images.

At present, there are many public pre-trained CNN models available for adopting to a specific task. These models include AlexNet [17], GoogLeNet [18], VGGNet [19], and ResNet [20]. These models are called a transfer learning such that the parameter learning for a new problem does not have to start from scratch, ones can apply the pre-train CNN to quickly learn a suitable parameter [21].

The pre-trained CNN method has two major differences from the train-from-scratch method. First, this method uses the transfer learning concept, thus the pre-trained method requires images for training significantly less than the train-from-scratch method. Second, the pre-trained method spends shorter time in training compared to the train-from-scratch. However, the pre-trained method has some limitations, that are, the accuracy of the model and size of images (pixel $\times$ pixel) depend on the pre-trained model.

To further speed up the pre-trained CNN, feature extraction is another important technique that should be applied. It is a process of reducing the dimensions of large quantities of information to a smaller extent that can represent the original data [22].

In this research work, we consider applying the pre-train $\mathrm{CNN}$ to the skin cancer classification. The traditional method of initial clinical screening for classifying the type of skin disease uses visual diagnosing of which required specialized expertise. The confirmation of diagnosis requires so many steps of clinical processes. Therefore, we propose an automatic approach applied for this classification task to help dermatologists for speeding up an early detection of skin cancer. Our automatic detection method for skin disease is based on deep learning model of CNN. Besides adopting the pre-trained $\mathrm{CNN}$, we consider a method to increase the classification performance by employing both image data and background knowledge of the patient in the modeling process.

\section{B. Literature Review}

There are many research works appeared in the literature that applied machine learning techniques to the dermatological domain. These works include the establishment of grading criteria for acne severity [23]. This study used the numbers of inflammatory eruptions (papules plus pustules) of half of the face to decide the correct classification among the four levels of acne severity: mild, moderate, severe, and very severe. Other research team [24] studied prediction of different dermatological conditions using naïve Bayesian classification. This research used medical data set containing records of 230 patients with 21 medical attributes for creating model to predict the probability of occurring eight dermatological conditions: scarlet fever, rubella, measles, fifth disease, chickenpox, entrovirus, no vaccination subitum, and Kawasaki. Recent research work [25] performed dermatologist-level 
classification of skin cancer using deep neural networks. The dataset used in this work was images from Stanford Hospital. The researchers create model by pre-trained CNN model of Google's Inception v3 using transfer learning. The accuracy of model is $72.1 \pm 0.9 \%$ for 3-way classification and $55.4 \pm 1.7 \%$ for 9 -way classification.

In our work, we employ both image data and background knowledge of the patient for creating model to 7-way classification. Our research is different from the previous work [25] in that we apply both image data and background knowledge of each patient to create model.

\section{MOdel CREATION METHOD}

In this research, we design the process for creating and assessing the classification model for dermatological classification with deep learning technique. We employ $\mathrm{CNN}$ architecture of AlexNet with transfer learning scenario as the starting point. In order to broadly investigating the transfer-learning concept, we create three types of models as shown in Fig. 2. These three models are named as Alexnet-TL, FESVM, and FESVM+PD, respectively.

- Alexnet-TL is the CNN model built with the pre-trained Alexnet architecture that has been fine-tuned to fit the specific task of skin disease classification.

- FESVM is basically the pre-trained Alexnet-TL that has been used as a feature extraction to be classified further with the support vector machine (SVM). Feature extraction is added as a pre-processing step to speed up the model learning process.

- FESVM+PD is the extension of the FESVM method by adding other patient data as the background knowledge. Image data and background knowledge are then passed to the SVM modeling step.

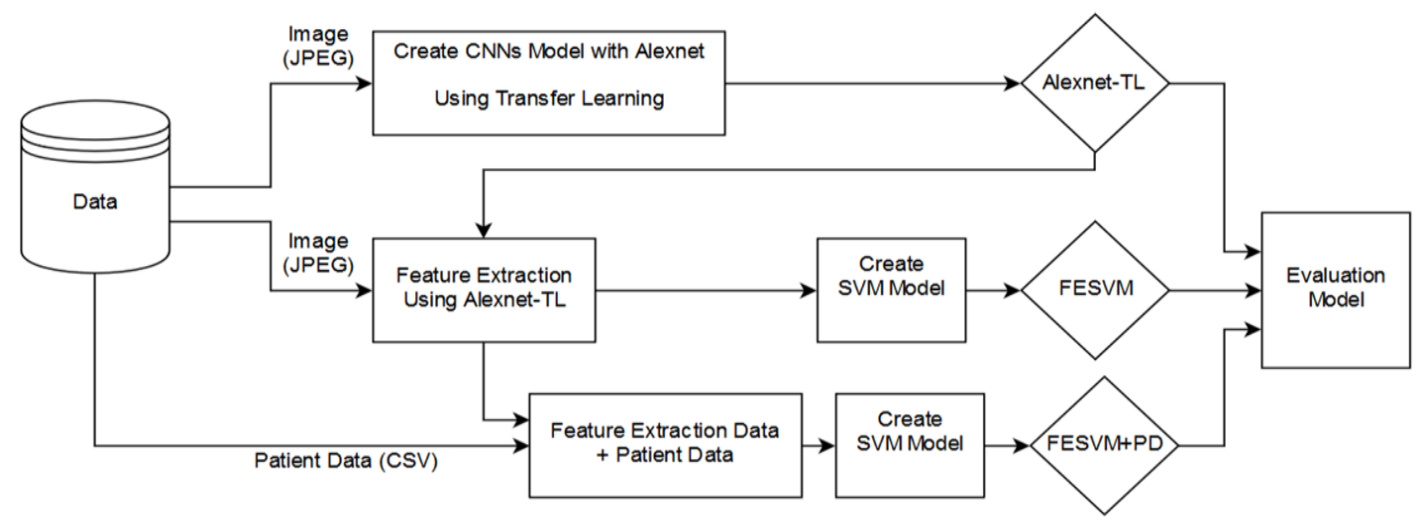

Fig. 2. The research framework proposed for the dermatological classification.

\section{A. Data}

This study uses the dermatological images from a public data source [26]. These data are the dermatoscopic images of seven skin lesions related to the disease. Sample pictures of each disease in all seven types are shown in Fig. 3.

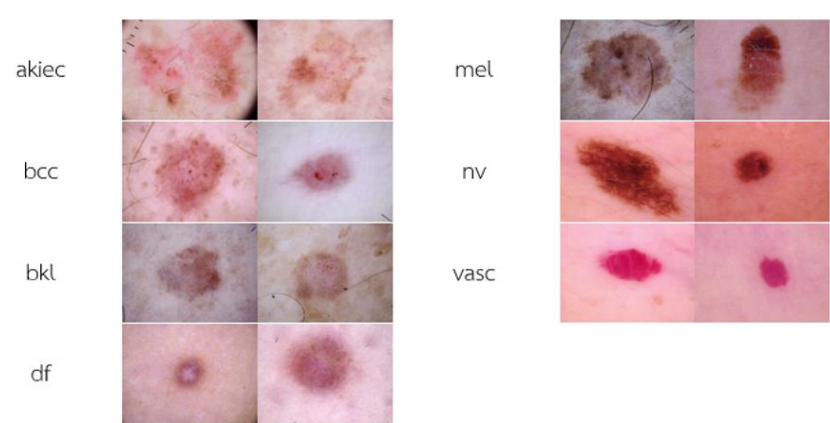

Fig. 3. Sample pictures of skin disease for all seven types.

TABLE I: THE TYPES OF SKIN DISEASE FROM A STUDIED DATASET AND NUMBER OF IMAGES FOR EACH TYPE

\begin{tabular}{lcc}
\hline \hline Disease & Acronym & Number \\
\hline Actinic keratoses and intraepithelial & akiec & 327 \\
$\quad$ carcinoma / Bowen's disease & & \\
Basal cell carcinoma & bcc & 514 \\
Benign keratosis-like lesions & bkl & 1,099 \\
Dermatofibroma & $\mathrm{df}$ & 115 \\
Melanoma & $\mathrm{mel}$ & 1,113 \\
Melanocytic nevi & $\mathrm{nv}$ & 6,705 \\
Vascular lesions & vasc & 142 \\
\hline \multicolumn{3}{c}{ Summation } \\
\hline
\end{tabular}

Table I shows the types of skin disease from a studied dataset and number of images for each type. In total, a dataset has 10,015 images. Most images are in a type of melanocytic nevi (nv), of which consists of 6,705 images.

This public dataset can be downloaded from a website "https://dataverse.harvard.edu". The data are dermatoscopic images that had been collected from different population of which acquired and stored by different modalities. The images are in JPEG format and attach the patient related data that we use as a background knowledge in our modeling step. The patient data are in CSV format. Data attribute details are summarized in Table II.

TABLE II: DETAILS OF PATIENT DATA USED AS BACKGROUND KNOWLEDGE IN OUR MOdELING PHASE

\begin{tabular}{ll}
\hline \hline Attribute name & Meaning \\
\hline image_id & Name of image \\
age & Age of patient \\
sex & Sex of patient \\
localization & The position of the disease on the body. \\
$\mathrm{dx}$ & Type of skin disease \\
\hline \hline
\end{tabular}

\section{B. Skin Diseases Classification}

In this research, we divide the images in a dataset into two subsets; one subset is used for training the model and the other subset is used for testing the model performance. The division ratio of training:testing in percentage is 70:30. We then apply the training data set for the 3 learning strategies (as shown in our research framework in Fig. 1) for skin 
diseases classification.

In our first strategy, we create CNNs model with Alexnet using transfer learning. It is the $\mathrm{CNN}$ model created by the pre-trained CNN model of Alexnet architecture using transfer learning by fine-tuning. The results from model creating is a classification model named Alexnet-TL (Alexnet with Transfer Learning)

The second strategy is applying the Alexnet-TL for the purpose of feature extraction. This strategy uses the previously created Alexnet-TL model for extracting the feature from the image data. It then employs SVM to classify the data obtained from the $\mathrm{CNN}$-based feature extractor. The model created by this strategy is named FESVM (Feature Extraction and SVM classification).

For the last strategy, we create SVM model from both feature extraction data that extract from image and patient data that are used as a background knowledge. This strategy employs SVM classifier for classification the skin diseases of patients on the same way as the second strategy. However, there are differences on the data used for modeling. This model will also consider additional patient data. The model is then named as FESVM + PD (Feature Extraction and SVM Classification + Patient Data). For this strategy, we design modeling step further to obtain three models (Type I, Type II, and Type III) depending on which details of patient data are considered. Details of patient data considered in each type are as follows:

Type I: Add age of patient only.

Type II: Add age and sex of patient.

Type III: Add age, sex, and position of the disease on the body of patient.

\section{EXPERIMENTAL RESULTS}

We used overall accuracy as a metric for evaluating the performance of the models to assess the correctness of skin disease classification. After performing experimentation according to each strategy (Alexnet-TL, FESVM, FESVM+PD: Type I, II, III), classification performances of all models are compared. The results are summarized in Table III.

From Table III, it can be seen that the best accuracy is from the FESVM+PD model. It can classify the skin diseases more accurate than the FESVM model and the Alexnet-TL model. Among the three types of FESVM+PD method, the model from the type III shows the best performance. This means that modeling with image data incorporating with the patient's background knowledge for classifying skin disease, especially skin cancer, get higher accuracy than simply using the images. We also observe that more details of background knowledge result in better classification results.

TABle III: Comparative Results OF THE Model Performance

\begin{tabular}{lcc}
\hline \hline \multicolumn{1}{c}{ Model } & $\begin{array}{c}\text { Accuracy } \\
\text { (Training Data) }\end{array}$ & $\begin{array}{c}\text { Accuracy } \\
\text { (Testing Data) }\end{array}$ \\
\hline Alexnet-TL & $84.94 \%$ & $79.29 \%$ \\
FESVM & $100 \%$ & $78.70 \%$ \\
FESVM+PD (Type I) & $100 \%$ & $80.16 \%$ \\
FESVM+PD (Type II) & $100 \%$ & $80.16 \%$ \\
FESVM+PD (Type & $100 \%$ & $80.39 \%$ \\
III) & & \\
\hline \hline
\end{tabular}

The performance of each classification model when further categorized by each type of skin disease can be shown in a form of confusion matrix in Fig. 4 to Fig. 8. Fig. 4 shows performance of the Alexnet-TL model. Fig. 5 shows the performance of FESVM model. Fig. 6 to Fig. 8 are performances of the FESVM+PD models with Type I, II, and III, respectively.

On comparing performances of DL model built from the transfer learning strategy (Alexnet-TL model performance in Fig. 4) and the model built from the strategy that performs feature extraction with Alexnet-TL and then classify with SVM (FESVM model performance in Fig. 5), the overall accuracy of Alexnet-TL model (79.3\%) is a little bit better than the FESVM model 78.7\%. But the FESVM model can predict the four (out of seven) specific types of bcc, bkl, df, and mel diseases more accurate than the Alexnet-TL model, whereas the Alexnet-TL model is good at predicting the aklec, nv and vasc skin disease.

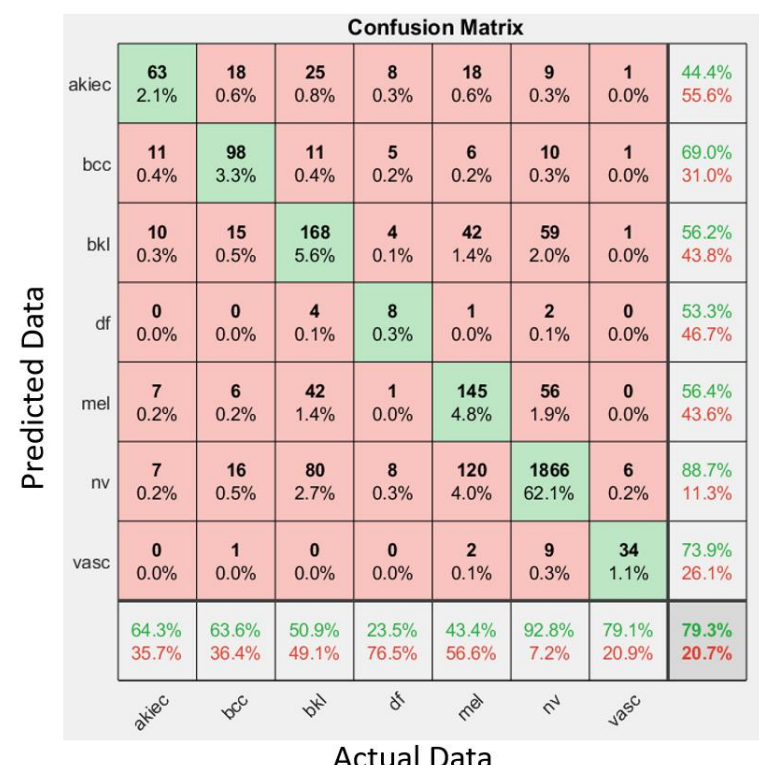

Fig. 4. Confusion matrix of Alexnet-TL model in test data.

\begin{tabular}{|c|c|c|c|c|c|c|c|c|}
\hline \multirow[b]{2}{*}{ akiec } & \multicolumn{8}{|c|}{ Confusion Matrix } \\
\hline & $\begin{array}{c}54 \\
1.8 \%\end{array}$ & $\begin{array}{c}11 \\
0.4 \%\end{array}$ & $\begin{array}{c}17 \\
0.6 \%\end{array}$ & $\begin{array}{c}5 \\
0.2 \%\end{array}$ & $\begin{array}{c}13 \\
0.4 \%\end{array}$ & $\begin{array}{c}8 \\
0.3 \%\end{array}$ & $\begin{array}{c}0 \\
0.0 \%\end{array}$ & $\begin{array}{l}50.0 \% \\
50.0 \%\end{array}$ \\
\hline bcc & $\begin{array}{c}15 \\
0.5 \%\end{array}$ & $\begin{array}{c}101 \\
3.4 \%\end{array}$ & $\begin{array}{c}8 \\
0.3 \%\end{array}$ & $\begin{array}{c}3 \\
0.1 \%\end{array}$ & $\begin{array}{c}7 \\
0.2 \%\end{array}$ & $\begin{array}{c}14 \\
0.5 \%\end{array}$ & $\begin{array}{c}\mathbf{5} \\
0.2 \%\end{array}$ & $\begin{array}{l}66.0 \% \\
34.0 \%\end{array}$ \\
\hline bkl & $\begin{array}{c}13 \\
0.4 \%\end{array}$ & $\begin{array}{c}19 \\
0.6 \%\end{array}$ & $\begin{array}{c}185 \\
6.2 \%\end{array}$ & $\begin{array}{c}2 \\
0.1 \%\end{array}$ & $\begin{array}{c}\mathbf{3 8} \\
1.3 \%\end{array}$ & $\begin{array}{c}66 \\
2.2 \%\end{array}$ & $\begin{array}{c}1 \\
0.0 \%\end{array}$ & $\begin{array}{l}57.1 \% \\
42.9 \%\end{array}$ \\
\hline df & $\begin{array}{c}\mathbf{2} \\
0.1 \%\end{array}$ & $\begin{array}{c}\mathbf{2} \\
0.1 \%\end{array}$ & $\begin{array}{c}\mathbf{4} \\
0.1 \%\end{array}$ & $\begin{array}{c}14 \\
0.5 \%\end{array}$ & $\begin{array}{c}1 \\
0.0 \%\end{array}$ & $\begin{array}{c}\mathbf{2} \\
0.1 \%\end{array}$ & $\begin{array}{c}0 \\
0.0 \%\end{array}$ & $\begin{array}{l}56.0 \% \\
44.0 \%\end{array}$ \\
\hline mel & $\begin{array}{c}6 \\
0.2 \%\end{array}$ & $\begin{array}{c}2 \\
0.1 \%\end{array}$ & $\begin{array}{c}\mathbf{4 3} \\
1.4 \%\end{array}$ & $\begin{array}{c}2 \\
0.1 \%\end{array}$ & $\begin{array}{c}155 \\
5.2 \%\end{array}$ & $\begin{array}{c}95 \\
3.2 \%\end{array}$ & $\begin{array}{c}0 \\
0.0 \%\end{array}$ & $\begin{array}{l}51.2 \% \\
48.8 \%\end{array}$ \\
\hline $\mathrm{nv}$ & $\begin{array}{c}8 \\
0.3 \%\end{array}$ & $\begin{array}{c}19 \\
0.6 \%\end{array}$ & $\begin{array}{c}73 \\
2.4 \%\end{array}$ & $\begin{array}{c}8 \\
0.3 \%\end{array}$ & $\begin{array}{c}119 \\
4.0 \%\end{array}$ & $\begin{array}{c}1822 \\
60.7 \%\end{array}$ & $\begin{array}{c}4 \\
0.1 \%\end{array}$ & $\begin{array}{l}88.7 \% \\
11.3 \%\end{array}$ \\
\hline vasc & $\begin{array}{c}\mathbf{0} \\
0.0 \%\end{array}$ & $\begin{array}{c}\mathbf{0} \\
0.0 \%\end{array}$ & $\begin{array}{c}\mathbf{0} \\
0.0 \%\end{array}$ & $\begin{array}{c}0 \\
0.0 \%\end{array}$ & $\begin{array}{c}\mathbf{1} \\
0.0 \%\end{array}$ & $\begin{array}{c}\mathbf{4} \\
0.1 \%\end{array}$ & $\begin{array}{c}33 \\
1.1 \%\end{array}$ & $\begin{array}{l}86.8 \% \\
13.2 \%\end{array}$ \\
\hline & $\begin{array}{l}55.1 \% \\
44.9 \%\end{array}$ & $\begin{array}{l}65.6 \% \\
34.4 \%\end{array}$ & $\begin{array}{l}56.1 \% \\
43.9 \%\end{array}$ & $\begin{array}{l}41.2 \% \\
58.8 \%\end{array}$ & $\begin{array}{l}46.4 \% \\
53.6 \%\end{array}$ & $\begin{array}{c}90.6 \% \\
9.4 \%\end{array}$ & $\begin{array}{l}76.7 \% \\
23.3 \%\end{array}$ & $\begin{array}{l}78.7 \% \\
21.3 \%\end{array}$ \\
\hline & $\partial x^{i e^{c}}$ & $0^{\circ}$ & $p^{t}$ & $\alpha^{4}$ & हे & त & $0^{0^{\circ}}$ & \\
\hline
\end{tabular}

Fig. 6 and Fig. 7 show the confusion matrices of the FESVM+PD method that employs both image data and 
patient data for modeling. Fig. 6 is the confusion matrix of the FESVM+PD (Type I) model that includes patient age, whereas Fig. 7 is the FESVM+PD (Type II) model that includes patient age and sex.

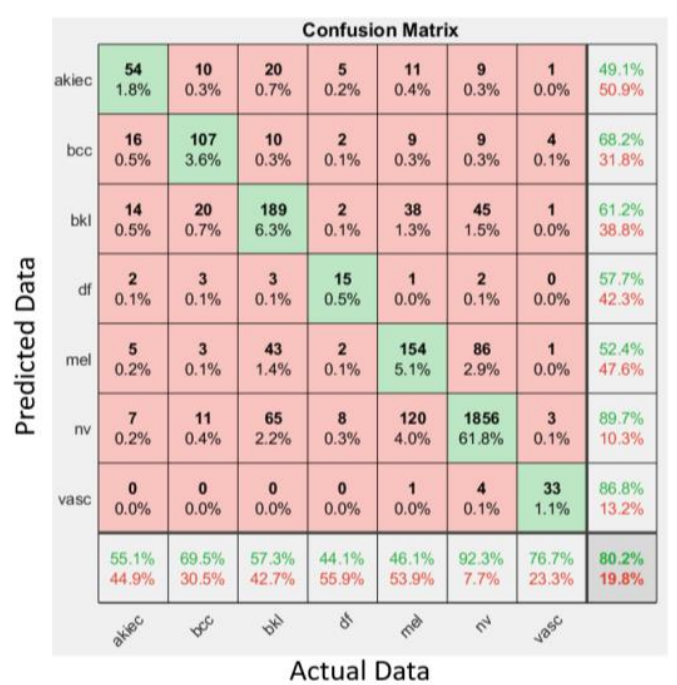

Fig. 6. Confusion matrix of FESVM+PD (Type I) model in test data.

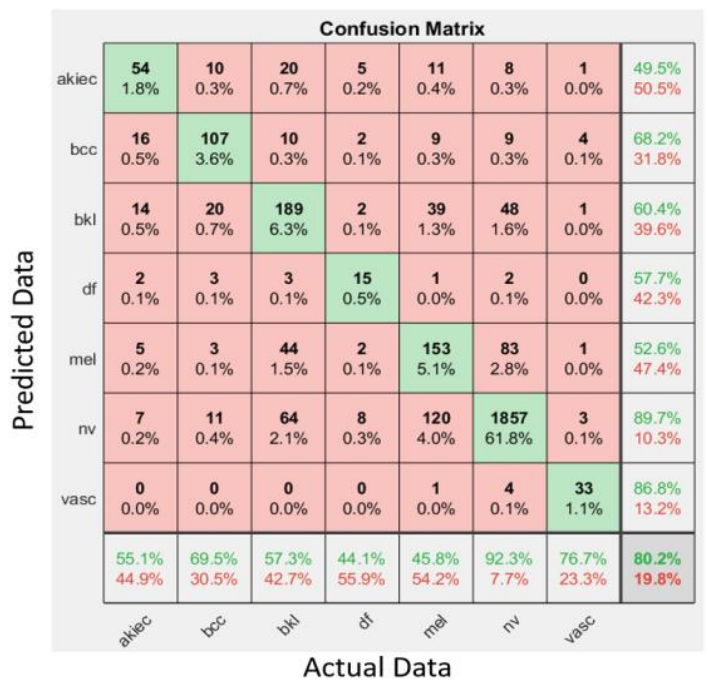

Fig. 7. Confusion matrix of FESVM+PD (Type II) model in test data.

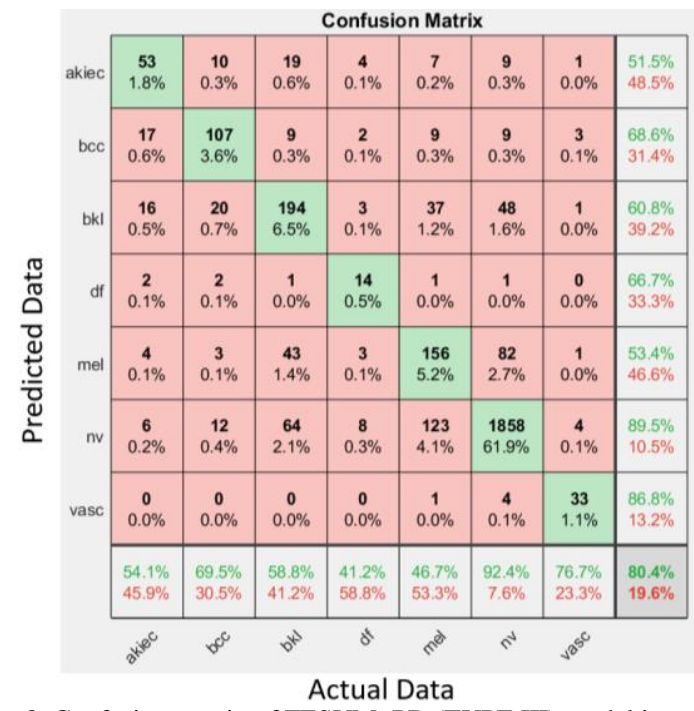

Fig. 8. Confusion matrix of FESVM+PD (TYPE III) model in test data.

The two types of FESVM+PD model can classify bcc, $\mathrm{bkl}, \mathrm{df}$, and mel diseases more accurate than the Alexnet-TL model with 41 and 45 patients for Type I and Type II, respectively. However, the Alexnet-TL model can classify akiec, nv and vasc disease more accurate than the FESVM+PD model (Type I and II) with 20 and 19 patients.

From the confusion matrix in Fig. 8, it can be noticed that the FESVM+PD (Type III) model can classify bcc, bkl, df, and mel diseases more accurate than the Alexnet-TL model with the amount of 52 patients. Moreover, the FESVM+PD (Type III) model can classify bkl, mel, and nv diseases better than the FESVM+PD (Type I and II) with the amount of 9 and 9 patients, respectively. However, the Alexnet-TL model can classify akiec, nv, and vasc diseases with better accuracy than the FESVM+PD (Type III) model on 19 patients.

\section{CONCLUSION}

Deep learning (DL) is currently a very accurate learning method on classifying images. There exist many powerful DL architectures ready for adoption to learn a new classification problem. Such availability provides the so called transfer learning in which other scientists do not need to build their own DL architecture from scratch. They instead apply the existing DL architecture with some fine-tuning on network parameters.

In this work, we present the DL transfer learning adoption strategy to be used for medical data classification. Our DL adoption strategy applies DL architecture to learn image data of skin diseases. Other patient data are also used as background knowledge in our learning strategy. On applying DL technique, we design three kinds of the learning models: transfer learning with the existing Alexnet architecture (called Alexnet-TL), SVM modeling from feature extraction data of image (FESVM), and SVM modeling from feature extraction data of image and patient data (FESVM+PD: Type I, II, III). The feature extraction of FESVM and FESVM+PD has been done by applying the Alexnet-TL strategy. That means we also use DL as a feature extractor.

We finally compare the performance of the three models by observing the overall accuracy. The experimental results show that SVM modeling from feature extraction data from image and patient data reveal the best performance. The FESVM+PD with Type III of adding background knowledge get the best performance as compared to other types of the FESVM+PD model. It can be concluded from the obtained results that the more available details of patient used as a background knowledge, the better accuracy obtained from the built model. Nevertheless, we hypothesize that the accuracy should also depend on the importance of the information to be added as a background knowledge. This hypothesis testing is thus our main subject for further investigation and research.

\section{CONFLICT OF INTEREST}

The authors declare no conflict of interest.

\section{AUTHOR CONTRIBUTIONS}

The first author is responsible for designing the research framework, organizing the experimentation steps and preparing the manuscript. The second author helps validating experimentation steps and confirming the results. The third author takes part in the experimentation design and 
programming work. The last author helps editing the manuscript and discussing the results.

\section{REFERENCES}

[1] R. S. Stern, "Prevalence of a history of skin cancer in 2007: Results of an incidence-based model," Archives of Dermatology, vol. 146, no. 3 , pp. 279-282, March 2010.

[2] H. W. Rogers, M. A. Weinstock, S. R. Feldman, and B. M. Coldiron, "Incidence estimate of nonmelanoma skin cancer (keratinocyte carcinomas) in the US population, 2012," JAMA Dermatology, vol. 151, no. 10, pp. 1081-1086, October 2015.

[3] Y. LeCun, Y. Bengio, and G. Hinton, "Deep learning," Nature, vol. 521, pp. 436-444, May 2015.

[4] A. Krizhevsky, I. Sutskever, and G. E. Hinton, "Imagenet classification with deep convolutional neural networks," Advances in Neural Information Processing Systems, pp. 1097-1105, 2012.

[5] C. Farabet, C. Couprie, L. Najman, and Y. LeCun, "Learning hierarchical features for scene labeling," IEEE Transactions on Pattern Analysis and Machine Intelligence, vol. 35, no. 8, pp. 1915-1929. August 2013.

[6] J. J. Tompson, A. Jain, Y. LeCun, and C. Bregler, "Joint training of a convolutional network and a graphical model for human pose estimation," Advances in Neural Information Processing Systems, pp. 1799-1807, 2014

[7] T. Mikolov, A. Deoras, D. Povey, L. Burget, and J. Černocký, "Strategies for training large scale neural network language models," in Proc. 2011 IEEE Workshop on Automatic Speech Recognition and Understanding (ASRU), December 2011, pp. 196-201.

[8] G. Hinton et al., "Deep neural networks for acoustic modeling in speech recognition: The shared views of four research groups," IEEE Signal Processing Magazine, vol. 29, no. 6, pp. 82-97, November 2012.

[9] S. Zaghbani, N. Boujneh, and M. S. Bouhlel, "Age estimation using deep learning," Computers \& Electrical Engineering, vol. 68, pp. 337-347, May 2018.

[10] M. K. Leung, H. Y. Xiong, L. J. Lee, and B. J. Frey, "Deep learning of the tissue-regulated splicing code," Bioinformatics, vol. 30, no. 12, pp. i121-i129, June 2014.

[11] H. Y. Xiong et al., "The human splicing code reveals new insights into the genetic determinants of disease," Science, vol. 347, no. 6218, article 1254806, Jan 2015.

[12] L. Wei, Y. Ding, R. Su, J. Tang, and Q. Zou, "Prediction of human protein subcellular localization using deep learning," Journal of Parallel and Distributed Computing, vol. 117, pp. 212-217, July 2018.

[13] D. Wang, A. Khosla, R. Gargeya, H. Irshad, and A. H. Beck, "Deep learning for identifying metastatic breast cancer," arXiv preprint arXiv:1606.05718, Jun 2016.

[14] C. Zhang, I. Sargent, X. Pan, H. Li, A. Gardiner, J. Hare, and P. M. Atkinson, "Joint deep learning for land cover and land use classification," Remote Sensing of Environment, vol. 221, pp. 173-187, February 2019.

[15] A. Gibson, J. Patterson, Deep Learning, O'Reilly Media, Inc., 2017, ch. 4.

[16] C. Y. Liou, W. C. Cheng, J. W. Liou, and D. R. Liou, “Autoencoder for words," Neurocomputing, vol. 139, pp. 84-96, September 2014.

[17] Y. LeCun, L. Bottou, Y. Bengio, and P. Haffner, "Gradient-based learning applied to document recognition," in Proc. the IEEE, November 1998, vol. 86, no. 11, pp. 2278-2324.

[18] C. Szegedy et al., "Going deeper with convolutions," in Proc. the IEEE Conference on Computer Vision and Pattern Recognition, 2015, pp. 1-9.

[19] K. Simonyan, and A. Zisserman, "Very deep convolutional networks for large-scale image recognition," arXiv preprint arXiv:1409.1556, April 2015.

[20] K. He, X. Zhang, S. Ren, and J. Sun, "Deep residual learning for image recognition," in Proc. the IEEE Conference on Computer Vision and Pattern Recognition, 2016, pp. 770-778.

[21] S. J. Pan and Q. Yang, "A survey on transfer learning," IEEE Transactions on Knowledge and Data Engineering, vol. 22, no. 10, pp. 1345-1359, October 2010.
[22] I. Guyon and A. Elisseeff, "An introduction to feature extraction," Feature Extraction, pp. 1-25, Springer, Berlin, Heidelberg, 2006.

[23] N. Hayashi, H. Akamatsu et al., "Establishment of grading criteria for acne severity," The Journal of Dermatology, vol. 35, no. 5, pp. 255-260, April 2008.

[24] K. K. Manjusha, K. Sankaranarayanan, and P. Seena, "Prediction of different dermatological conditions using naïve Bayesian classification," International Journal of Advanced Research in Computer Science and Software Engineering, vol. 4, no. 1, pp. 864-868, January 2014.

[25] A. Esteva, B. Kuprel, R. A. Novoa, J. Ko, S. M. Swetter, H. M. Blau, and S. Thrun, "Dermatologist-level classification of skin cancer with deep neural networks," Nature, vol. 542, no. 7639, pp. 115-118, June 2017.

[26] P. Tschandl, C. Rosendahl, and H. Kittler. (2018). The HAM10000 dataset, a large collection of multi-source dermatoscopic images of common pigmented skin lesions. Harvard dataverse. [Online]. Available:

https://dataverse.harvard.edu/dataset.xhtml?persistentId=doi:10.7910/ DVN/DBW86T

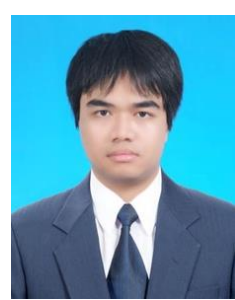

Kittipat Sriwong is currently a master student with the School of Computer Engineering, Institute of Engineering, Suranaree University of Technology, Thailand. He has been fully financial support by grant from Suranaree University of Technology throughout his bachelor and master study. He is a research assistant in the Data and Knowledge Engineering Research Unit, SUT. His current research of interest includes data mining, support vector machine and deep learning techniques, statistical data mining, and data mining applications in medical science.

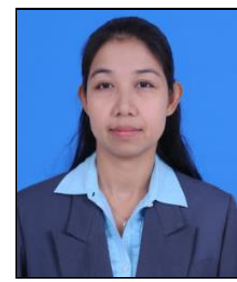

Supaporn Bunrit is a lecturer with computer Engineering School, SUT. She received her bachelor degree in science (mathematics) from Kasetsart University, Thailand, in 1997, master degree in science (computer science) from Chulalongkorn University, Thailand, in 2001. Her research of interest includes artificial neural network, deep learning, machine learning, digital image processing, computer vision, and time series analysis.

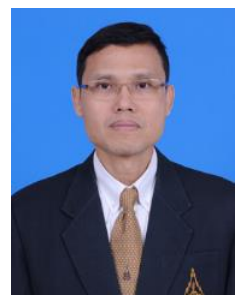

Kittisak Kerdprasop is an associate professor and the chair of the Computer Engineering School, Suranaree University of Technology, Thailand. He received his B.Ed. degree in mathematics from Srinakarinwirot University, Thailand, in 1986, M.S. in computer science from the Prince of Songkla University, Thailand, in 1991 and Ph.D. in computer science from Nova Southeastern University, U.S.A., in 1999. His current research includes machine learning, statistical data modeling, and artificial intelligence.

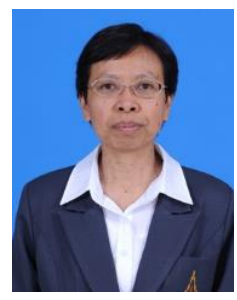

Nittayak Kerdprasop is an associate professor and the head of the Data and Knowledge Engineering Research Unit, School of Computer Engineering, Suranaree University of Technology, Thailand. She received her B.S. degree in radiation techniques from Mahidol University, Thailand, in 1985, M.S. in computer science from the Prince of Songkla University, Thailand, in 1991 and Ph.D. in computer science from Nova Southeastern University, U.S.A., in 1999. Her current research includes data mining, business intelligence, computational modeling in health and environmental sciences. 
Artificial Intelligence and Intelligent Systems 
